\title{
Evaporation of hot jupiters and hot neptunes
}

\author{
D. Ehrenreich ${ }^{1}$
}

\section{${ }^{1}$ Laboratoire d'Astrophysique de Grenoble [david.ehrenreich@obs.ujf-grenoble.fr]}

\begin{abstract}
Among the nearly five hundred extra-solar planets known, almost $30 \%$ orbit closer than 0.1 AU from their parent star. We will review the observations and the corresponding models of the evaporation of these 'hot jupiters'. The observations started with the discovery made with HST that the planet orbiting HD 209458 has an extended atmosphere of escaping hydrogen. Subsequent observations obtained with $H S T /$ STIS and $H S T /$ ACS confirm the escape of the gas. Even more, oxygen and carbon have been shown to be present at very high altitude in the upper atmosphere. Observations of other targets like HD 189733 and WASP-12 show that evaporation is a general phenomenon which could contribute to the evolution of planets orbiting close to their parent stars. To interpret these observations, we developed models to quantify the escape rate from the measured occultation depths. Numerous models have also been published to investigate mechanisms which can lead to the estimated escape rate. In general, the high temperature of the upper atmosphere heated by the far and extreme UV combined with the tidal forces allow a very efficient evaporation of the upper atmosphere. We will review the different models and their implications.
\end{abstract}

\section{Introduction}

Planetary transits are golden opportunities to probe the atmospheres of extrasolar planets. The atmospheric signals obtained with this method are usually tenuous, and few of them are firmly established. One of the strongest case is the detection in the visible of neutral atomic sodium in the hot jupiter HD 209458b. The sodium signature, a mere $\sim 2 \times 10^{-4}$ absorption, was obtained both from medium- to high-resolution, space-born and ground-based spectrographs (Charbonneau et al. 2002; Snellen et al. 2008) and secured by several independent data analyses (e.g., Sing et al. 2008a,b). In the ultraviolet (UV), planetary transits of HD 209458b have produced several remarkably large spectroscopic signatures, which are seen as absorptions in the emission lines originating from the stellar chromosphere (see Ehrenreich 2010 for a review). These large UV signatures, compared to the visible transit of the whole planet (1.6\%; Charbonneau et al. 2000; Henry et al. 2000), require the presence of an extended and evaporating H u upper atmosphere, or exosphere, to the planet. Similar UV observations have been recently carried out on different planets to find out whether atmospheric evaporation is a property common among hot jupiters. In this paper, I review the new results obtained on exoplanets HD 189733b and WASP-12b, as well as new high-resolution observations of the exospheric signature of HD 209458b. Then, I describe what sort of signature could

This is an Open Access article distributed under the terms of the Creative Commons Attribution-Noncommercial License 3.0, which permits unrestricted use, distribution, and reproduction in any noncommercial medium, provided the original work is properly cited. 
be expected for planets less massive than hot jupiters, such as the neptunian planet GJ 436b.

\section{Observations of the exospheres of extrasolar planets}

\subsection{Lyman- $\alpha$ transits of hot jupiters}

The strongest atmospheric signature observed for an exoplanet was obtained during the transit of the prototypical hot jupiter HD 209458b. It is a $(15 \pm 4) \%$ absorption in the stellar Lyman- $\alpha(\mathrm{Ly} \alpha)$ emission line of neutral atomic hydrogen $(\mathrm{HI})$. This result was obtained with medium-resolution spectra taken with the Space Telescope Imaging Spectrograph (STIS) on the Hubble Space Telescope (HST) (Vidal-Madjar et al. 2003). It was later confirmed from new observations at lower resolution from STIS (Vidal-Madjar et al. 2004) and the Advanced Camera for Surveys (ACS) on HST (Ehrenreich et al. 2008). It was also strengthened by independent data analysis (Ben-Jaffel 2007, 2008; see Vidal-Madjar et al. 2008). The large absorption requires the presence of an extended H I upper atmosphere, or exosphere, to the planet. This envelope is necessarily evaporating because (i) it must fill the planetary Roche lobe to account for the size of the absorptions, and (ii) the $\mathrm{H}$ I atoms must be accelerated by the stellar radiation pressure beyond the escape velocity of the planet to account for the absorption in the wings of the Ly $\alpha$ line (Vidal-Madjar et al. 2003). New cases of atmospheric escape have been recently reported for two hot jupiters. Lecavelier des Etangs et al. (2010) use HST/ACS to detect the H I exosphere of HD 189733b at Ly $\alpha$. Figure 1 compiles all Ly $\alpha$ transit light curves obtained so far, for HD 209458b and HD 189733b. Finally, Fossati et al. (2010) present observations of the highly irradiated planet host star WASP-12 with the Cosmic Origins Spectrograph (COS) on board HST, and interpret the tentative detection of extra absorptions in the metal lines of the star during the transit as signs of mass loss. Thus, atmospheric evaporation could be a common phenonemon among close-in planets.

Modelling Ly $\alpha$ transit light curve, as done in Fig. 1 allows the mass loss rate $(\dot{m})$ and the stellar high-energy flux $\left(F_{\mathrm{X} / \mathrm{EUV}}\right)$ to be constrained. In the case of HD 189733b, Lecavelier des Etangs et al. (2010; see their Fig. 13) find $10^{10} \lesssim \dot{m} \lesssim 10^{11} \mathrm{~g} \mathrm{~s}^{-1}$ and $F_{\mathrm{X} / \mathrm{EUV}} \sim 20 \times$ the solar value. The mass loss rate is negligible regarding the total mass of the planet, yet it has a dramatic impact on the observable size of the planet's upper atmosphere, for increasing values of $\dot{m}$.

\subsection{Heavy elements in the exosphere of HD 209458b}

The presence of elements heavier than hydrogen $(\mathrm{C}$ and $\mathrm{O})$ at high altitudes has been surmised from unresolved HST/STIS observations (Vidal-Madjar et al. 2004). Their presence requires an hydrodynamic escape (or blow-off) of the upper atmosphere, with the flow of escaping H I carrying heavier species up to the Roche limit (Vidal-Madjar et al. 2004; Linsky et al. 2010), where they are swept away by radiation pressure (Lecavelier des Etangs et al. 2004). The tentative detections presented in Vidal-Madjar et al. (2004) were retrieved in an independent analysis of the same data set (Ben-Jaffel \& Sona Hosseini 2010). The detection of C II was recently confirmed at a higher signalto-noise level by new observations of the Cosmic Origins Spectrograph (COS) on HST (Linsky et al. 2010), shown in Fig. 2. These authors additionally report an absorption in the emission line of ionized silicon (Si III) that had not been seen previously. Assuming hydrodynamic model values for the gas temperature, outflow velocity at the limb of the outflow as seen in the C II lines, and solar abundances, Linsky et al. (2010) find 
mass-loss rates in the range $(8-40) \times 10^{10} \mathrm{~g} \mathrm{~s}^{-1}$.

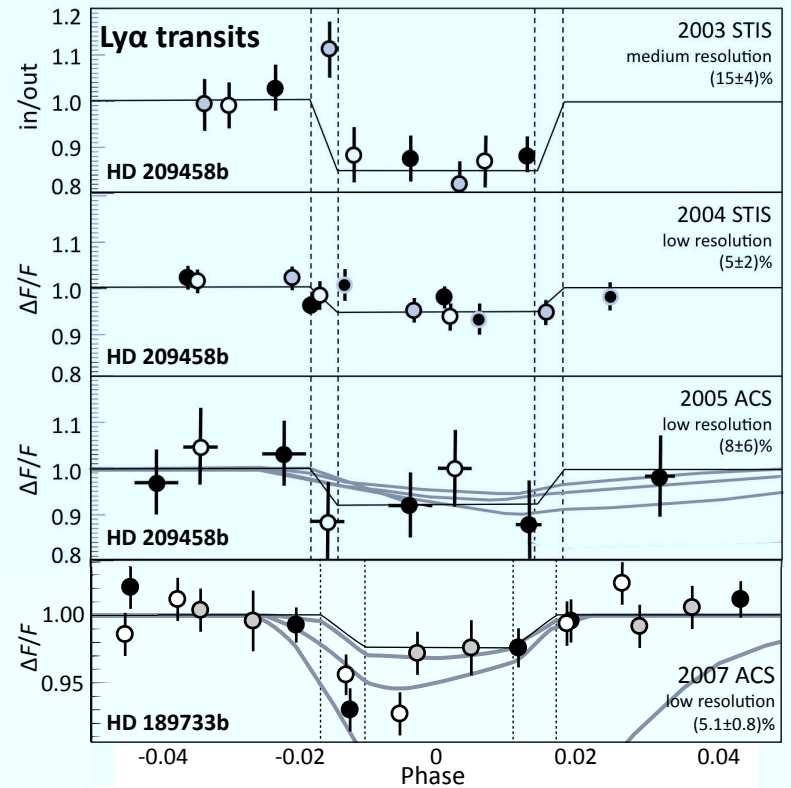

Figure 1: Lyman- $\alpha$ transit light curves obtained for HD $209458 b$ ((a)Vidal-Madjar et al. 2003, (b) Vidal-Madjar et al. 2004, and (c) Ehrenreich et al. 2008) at different resolutions and HD 1897336 ((d) Lecavelier des Etangs et al. 2010). Different colors indicate different transits. Gray curves are model light curves produced by particle simulations of the atmospheric escape.

The more significant observing results are summarized in Table 1 for each species and planet. These results have initiated a comprehensive modeling effort from different groups (e.g., Lecavelier des Etangs et al. 2004; Lecavelier des Etangs 2007; Lecavelier des Etangs, Vidal-Madjar, \& Désert 2008; Yelle 2004, 2006; Tian et al. 2005; GarcíaMuñoz 2007; Schneiter et al. 2007; Hubbard et al. 2007; Penz et al. 2008; Davis \& Wheatley 2009; Murray-Clay, Chiang \& Murray 2009). The global picture given by the above-cited models suggests that the upper atmospheres of close-in giant planets must be heated by the stellar X-rays, extreme and far UV, to about $10000 \mathrm{~K} .{ }^{1}$ The canonical atmospheric mass loss rate $\dot{m}$ derived by these models is between $10^{10}$ and $10^{11} \mathrm{~g} \mathrm{~s}^{-1}$. In this case, evaporation should not severely impact the life of observed giant planets. Meanwhile, it could be more critical for lower-mass planets such as hot neptunes.

\section{Evaporation of hot neptunes}

I use the case of GJ 436b, the first transiting neptune-mass exoplanet, to illustrate how we can predict the observable depth of the UV transit, i.e., the size of the signature

${ }^{1}$ A similar temperature has been actually measured in the thermosphere of HD 209458b (Ballester, Sing, \& Herbert 2007) 

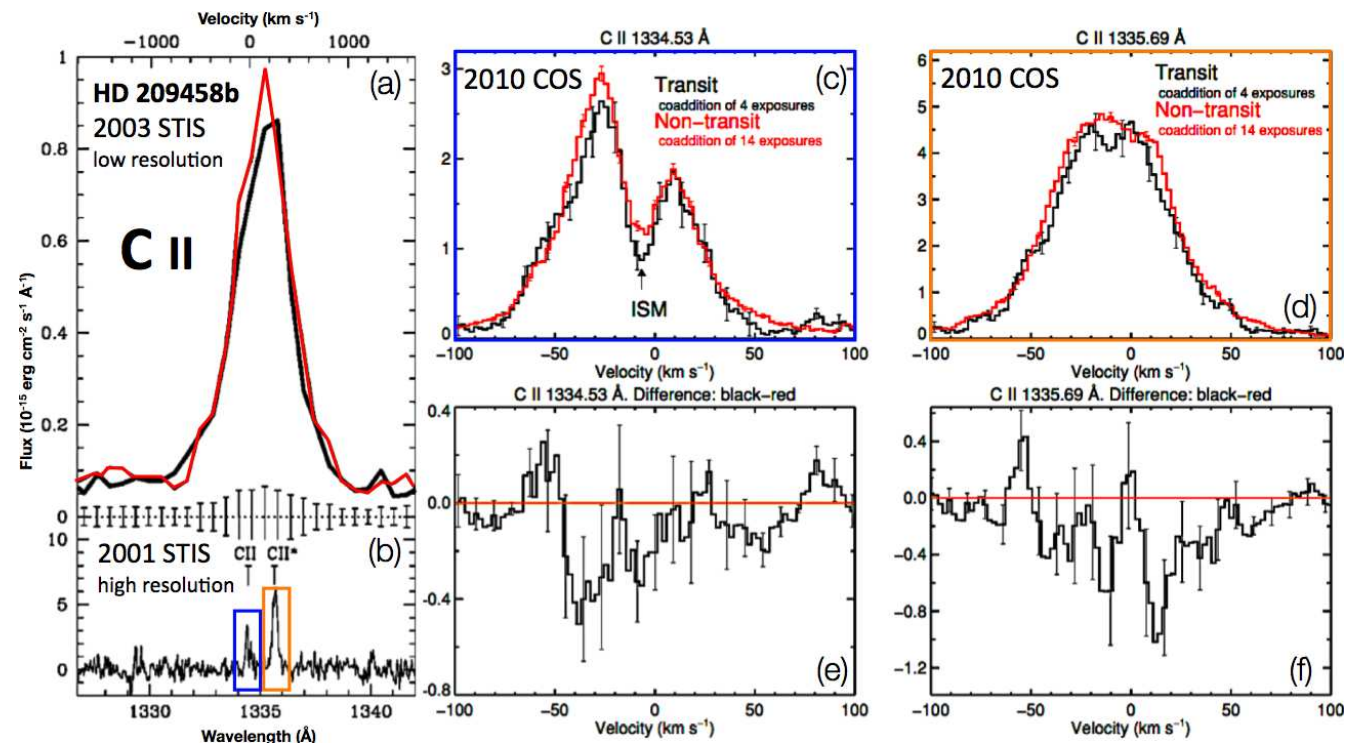

Figure 2: In-transit absorptions in the CII doublet. (a) Low-resolution data of the with the in-transit (black) and out-of-transit (red) stellar emission (Vidal-Madjar et al. 2004). (b) High-resolution STIS spectrum. (c,d) High-resolution COS spectra of both components of the $C$ II doublet. (e,f) Subtractions of the black and red curves from panel (c) and (d), showing the velocity structure in the exospheric absorptions

of the planet's upper atmosphere in transmission spectroscopy. Various models (e.g., Figueira et al. 2009) predict an hydrogen envelope for this planet, based on its location in a mass-radius diagram. GJ 436b lies significantly above the mass-radius relation for a planet composed by $100 \%$ water (Gillon et al. 2007; see their Fig. 3). Ultraviolet transmission spectroscopy could be used to detect the hydrogen envelope.

Table 1: Exoplanet atmospheric detections from UV transits

\begin{tabular}{cccccc}
\hline Species & Resolved? & $\Delta F / F$ & $\begin{array}{c}\text { transit } \\
\text { detection }\end{array}$ & $\begin{array}{c}\text { atmosphere } \\
\text { detection }\end{array}$ & Refs. \\
\hline \multicolumn{7}{c}{ HD 209458b } \\
H I & Yes & $15 \pm 4 \%$ & $3.8 \sigma$ & $3.4 \sigma$ & $(1)$ \\
O I & No & $12.8 \pm 4.5$ & $2.8 \sigma$ & $2.5 \sigma$ & $(2)$ \\
C II & Yes & $7.8 \pm 1.3$ & $6 \sigma$ & $4.8 \sigma$ & $(3)$ \\
Si III & Yes & $8.2 \pm 1.4$ & $5.9 \sigma$ & $4.7 \sigma$ & $(3)$ \\
\hline \multicolumn{7}{c}{ HD 189733b } \\
H I & No & $5.1 \pm 0.8$ & $6.4 \sigma$ \\
\hline \multicolumn{7}{c}{ WASP-12b } \\
Mg II & No & $3.0 \pm 0.5$ & $6 \sigma$ & $3.4 \sigma$ & $(4)$ \\
\hline
\end{tabular}

References. (1) Vidal-Madjar et al. 2003; (2) Vidal-Madjar et al. 2004; (3) Linsky et al. 2010; (4) Lecavelier des Etangs et al. (2010);

(5) Fossati et al. (2010). 


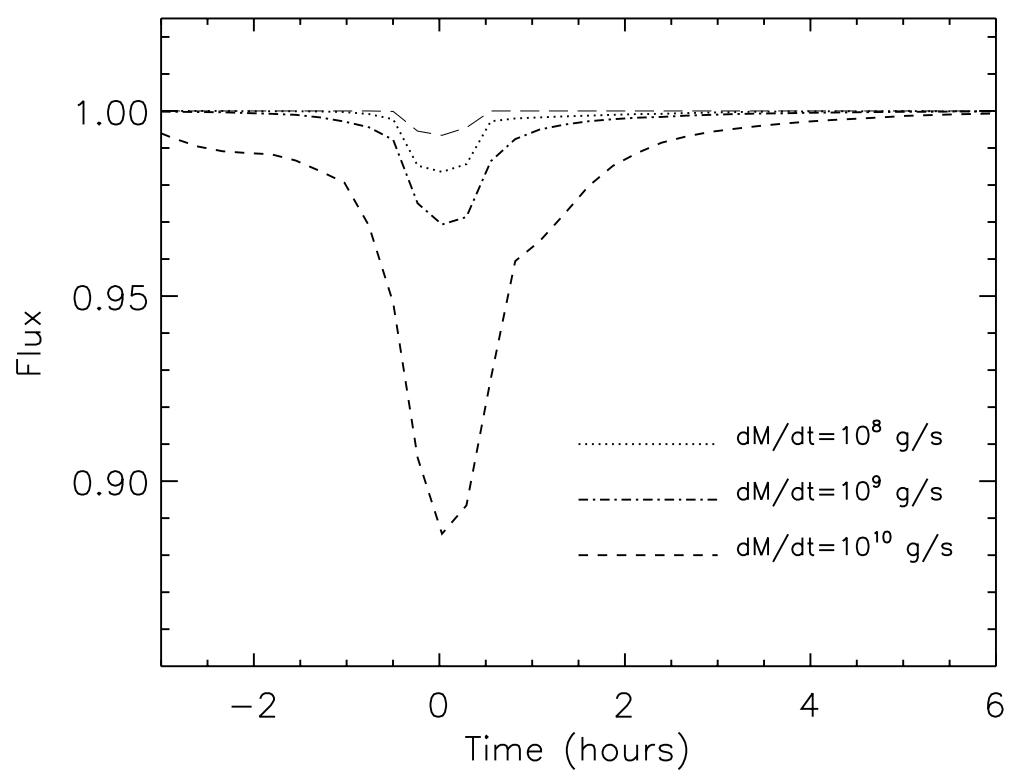

Figure 3: Predicted Lya transit light curves for the hot neptune GJ 436b, assuming different possible values of the mass loss rate. From Ehrenreich, Lecavelier des Etangs 85 Delfosse (submitted).

The framework is that of an energy-limited atmospheric escape, where the mass loss rate is approximately proportional to the amount of energy radiated from the star at short wavelengths (X/EUV). In fact, this radiated energy is used to overcome the potential gravitational energy of the planet (which includes the tidal forces resulting from the proximity of the star), following $-\mathrm{dE}_{\text {pot }}^{\prime}=\eta \mathrm{dE}_{\mathrm{X} / \mathrm{EUV}}$. The $\eta$ factor accounts for the heating efficiency of the planet's thermosphere by high-energetic radiation. So far, this factor is still unconstrained for extrasolar planets. Following Lecavelier des Etangs (2007), it is possible to calculate for each transiting planet - and for a given value of $\eta$ - an atmospheric mass loss rate $\dot{m}$. For GJ $436 \mathrm{~b}, \dot{m}$ is estimated between $10^{8}$ and $10^{10} \mathrm{~g} \mathrm{~s}^{-1}$ for $\eta$ between 0.01 and 1 , respectively (Ehrenreich \& Désert, submitted).

To calculate a theoretical set of $\mathrm{H}$ I transit light curves for this range of mass-loss rates, one needs to know the Ly $\alpha$ brightness of the star. Ehrenreich, Lecavelier des Etangs, \& Delfosse (submitted) present a HST/STIS spectrum of the M dwarf GJ 436 at Ly $\alpha$. From this observation, they derive a Ly $\alpha$ flux of $\sim 2 \times 10^{-13} \mathrm{erg} \mathrm{s}^{-1} \mathrm{~cm}^{-2}$. They use this information to predict Ly $\alpha$ transit light curves for different $\dot{m}$, as shown in Fig. 3. The depth of the obtained Ly $\alpha$ light curve $\left(\sim 11 \%\right.$ for $\left.\dot{m} \sim 10^{10} \mathrm{~g} \mathrm{~s}^{-1}\right)$ will allow the hydrogen envelope to be detected with HST. This prediction also opens new perspectives for the atmospheric characterization of other hot neptunes and even for the atmospheric detection of hot super-earths.

Acknowledgements. I thank the organisers of the Colloquium for an excellent meeting. I acknowledge financial support from the Centre National d'Études Spatiales (CNES). 


\section{EPJ Web of Conferences}

\section{References}

Ballester, G. E., Sing, D. K., Herbert, F. 2007, Nature , 445, 511

Ben-Jaffel, L. 2007, ApJ, 671, L61

Ben-Jaffel, L. 2008, ApJ, 688, 1352

Ben-Jaffel, L. \& Sona-Hosseini, S. 2010, ApJ, 709, 1284

Charbonneau, D., Brown, T. M., Latham, D. W., \& Mayor, M. 2000, ApJ, 529, L45

Charbonneau, D., Brown, T. M., Noyes, R. W., \& Gilliland, R. L. 2002, ApJ, 568, 377

Davis, T. A., \& Wheatley, P. J. 2009, MNRAS, 396, 1012

Ehrenreich, D. et al. 2008, A\&A, 483, 933

Ehrenreich, D. 2010, in EAS Publication Series, 41, Physics and Astrophysics of Planetary

Systems, eds. T. Montmerle, D. Ehrenreich, A.-M. Lagrange, 429

Ehrenreich, D., Désert, J.-M. 2010, A\&A, submitted

Ehrenreich, D., Lecavelier des Etangs, \& A., \& Delfosse, X. 2010, A\&A, submitted

Fossati, L. et al. 2010, ApJ, 714, L222

Henry, G. W., Marcy, G. W., Butler, R. P., \& Vogt, S. S. 2000, ApJ, 529, L41

Hubbard, W. B. et al. 2007, Icarus, 187, 358

Lecavelier des Etangs, A. et al. 2010, A\&A, 514, A72

Lecavelier des Etangs, A. 2007, A\&A, 461, 1185

Lecavelier des Etangs, A. et al. 2004, A\&A, 418, L1

Lecavelier des Etangs, A. Vidal-Madjar, A., Désert, J.-M. 2008, Nature, 456, E1 Linsky, J. et al. 2010, ApJ, 717, 1291

Murray-Clay, R. A., Chiang, E. I., Murray, N. 2009, ApJ, 693, 23

Penz, T., Micela, G., Lammer, H. 2008, A\&A, 477, 309

Schneiter, E. M. et al. 2007, ApJ, 671, L57

Sing, D. K. et al. 2008a, ApJ, 686, 667

Sing, D. K. et al. 2008b, ApJ, 686, 658

Snellen, I. A. G. et al. 2008, A\&A, 487, 357

Tian, F. et al. 2005, ApJ, 621, 1049

Vidal-Madjar, A. et al. 2003, Nature , 422, 143

Vidal-Madjar, A. et al. 2004, ApJ, 604, L69

Vidal-Madjar, A. et al. 2008, ApJ, 676, L57

Yelle, R. 2004, Icarus, 170, 167

Yelle, R. 2006, Icarus, 183, 508 